\title{
Search for universality in one-dimensional ballistic annihilation kinetics.
}

\author{
Pierre-Antoine Rey and Michel Droz \\ Département de Physique Théorique, Université de Genève, CH-1211 Genève 4, Switzerland. \\ Jarosław Piasecki \\ Institute of Theoretical Physics, Warsaw University, Hoża 69, Pl-00 681 Warsaw, Poland
}

\section{UGVA-DPT 1997/07-983}

(March 22, 2018)

\begin{abstract}
We study the kinetics of ballistic annihilation for a one-dimensional ideal gas with continuous velocity distribution. A dynamical scaling theory for the long time behavior of the system is derived. Its validity is supported by extensive numerical simulations for several velocity distributions. This leads us to the conjecture that all the continuous velocity distributions $\phi(v)$ which are symmetric, regular and such that $\phi(0) \neq 0$ are attracted in the long time regime towards the same Gaussian distribution and thus belong to the same universality class. Moreover, it is found that the particle density decays as $n(t) \sim t^{-\alpha}$, with $\alpha \simeq 0.785 \pm 0.005$.
\end{abstract}

PACS numbers: 82-20.Mj, 05.20Dd

\section{INTRODUCTION}

Ballistically-controlled reactions provide simple examples of non-equilibrium systems with complex kinetics and have recently attracted a lot of interest [1 9]. They consist of an assembly of particles moving freely between collisions with given velocities. When two particles meet, they instantaneously annihilate each other and disappear from the system. In one dimension, it is enough to consider point particles and we shall restrict ourselves to this case here. The system with only two possible velocities $+c$ or $-c$ has been studied in a pioneering work by Elskens and Frisch [1]. Using combinatorial analysis, they showed that, in the long time limit, the density of particles was decreasing according to a power law $t^{-1 / 2}$ in the case of a symmetric initial velocity distribution; Krug and Spohn [2] obtained independently similar results. Later on, Redner et al. [3, [4] have studied the case of more general velocity distributions. Based on numerical simulations and mean-field type arguments they showed that the exponent characterizing the power law decay of the particle density could depend on the velocity distribution. The case of a general distribution has been recently approached analytically by Piasecki [5] and Droz et al. [6.6]. It was shown that the annihilation dynamics reduced exactly to a single closed equation for the two-particle conditional probability [5]. A method which permits to solve this evolution equation for discrete velocity distributions was developed in [6] and explicitly applied to the case of a symmetric three-velocity distribution. It turns out that on one hand, different dynamical behaviors can occur depending on the relative probability weights given to the three velocities and, on the other hand, that the fluctuations are playing a very important role, invalidating the predictions of mean-field or Boltzmann like approaches.

The case of continuous velocity distributions was considered first by Ben-Naim and co-workers [3]. Within a
Boltzmann approximation, they showed that the particle density was decreasing according to a power law $t^{-\alpha}$. Moreover, for initial velocity distributions with a powerlaw dependence near the origin and a cut-off at $v_{0}$, i.e. $\phi(v) \sim|v|^{\mu} \theta\left(v_{0}-|v|\right)(-1<\mu<0)$, they found that the exponent $\alpha$ was non-universal and was depending continuously on the value of $\mu$. This dependence was confirmed by Monte-Carlo simulations. Note that the particular case $\mu=0$ corresponds to a uniform distribution. In this case, and in one dimension, numerical integration of the Boltzmann equation led to $\alpha=0.77$, while Monte-Carlo simulations gave $\alpha=0.76$. These values are very different from the ones obtained for the discrete two- and three-velocity distributions.

The distributions considered by Redner et al. form a very special subset of the continuous velocity distributions. So, it would not be justified to generalize their conclusions. A deeper understanding of the problem requires to study a wider class of distributions. This was our motivation for this work. Several questions could be asked. The main issue analyzed here, concerns the existence of universality classes in this problem. Namely, does it exist a class of continuous velocity distributions for which all its members possess the same exponent $\alpha$, and what are the characteristics of this class? As we have seen above, to approach this question it is not sufficient to consider a mean-field like approximation, but it is necessary to build up a theory taking into account the fluctuations.

This paper is organized as follows. In Section II, we define the model and present the main ideas and conclusions obtained within the analytical approach developed by Piasecki [5] and Droz et al. [6.,7]. Based on those results a dynamical scaling theory is developed in Section III, whose validity is confirmed by extensive numerical simulations in Section IV. Three different continuous velocity distributions (Gaussian, uniform and Lorentzian) are studied. We found that they are all attracted dur- 
ing the time evolution towards a Gaussian distribution for which the decay exponent is $\alpha=0.785 \pm 0.005$. It is conjectured that all the continuous velocity distributions regular near the origin and having a finite nonzero value for $v=0$ are attracted by the same Gaussian distribution and thus belong to the same universality class. Moreover, it is shown why the velocity distributions with a power-law dependence with negative exponent $\mu$ near the origin are not attracted by the Gaussian distribution and can lead to non-universal exponents. Final remarks are made in Section $\mathrm{M}$ and the numerical algorithm used in the simulations is explained in Appendix.

\section{THE MODEL}

We assume that initially the particles are uniformly distributed in space, according to the Poisson law, without any correlations between their velocities. Note that other distributions than Poisson could be considered as long as one is dealing with a renewal process (see 10 for the definition of a renewal process). The fundamental role in the analysis made in [5, 6] is played by the distribution of nearest neighbors. Suppose that at time $t$ there is a particle at point $x_{1}$ in the fluid, moving with velocity $v_{1}$. We denote by

$$
\mu\left(x_{2}, v_{2} \mid x_{1}, v_{1} ; t\right)
$$

the conditional probability density for finding its right nearest neighbor at distance $x_{21}=x_{2}-x_{1}>0$, with velocity $v_{2}$. The density (11) satisfies the normalization condition

$$
\int d 2 \mu(2 \mid 1 ; t)=1
$$

where a convenient short hand notation

$$
j \equiv\left(x_{j}, v_{j}\right), \quad d j \equiv d x_{j} d v_{j}, \quad j=1,2, \ldots
$$

has been used. As a rigorous consequence of the dynamics of ballistic annihilation [5, 6], this quantity obeys the equation:

$$
\begin{aligned}
& {\left[\frac{\partial}{\partial t}+v_{1} \frac{\partial}{\partial x_{1}}+v_{2} \frac{\partial}{\partial x_{2}}+C(1,2)\right] \mu(2 \mid 1 ; t)} \\
& =\int d 3[C(1,3) \mu(3 \mid 1 ; t)-C(2,3) \mu(3 \mid 2 ; t)] \mu(2 \mid 1 ; t) \\
& \quad+\int d 3 \int d 4 C(3,4) \mu(3 \mid 1 ; t) \mu(4 \mid 3 ; t) \mu(2 \mid 4 ; t) .
\end{aligned}
$$

where $C(1,3)$ is the binary collision operator defined as:

$$
C(1,2)=v_{12} \theta\left(v_{12}\right) \delta\left(x_{21}-0^{+}\right)
$$

$\theta(x)$ is the usual Heaviside function and $v_{12}$ stands for $v_{1}-v_{2}$. If each particle has initially the same continuous probability density $\phi(v)$ to move with velocity $v$, the state of the system is translationally invariant. As a consequence, $\mu(2 \mid 1 ; t)$ depends in the position space only on the distance $x_{21}$ [we shall then write $\left.\mu\left(x_{2}, v_{2} \mid x_{1}, v_{1} ; t\right)=\mu\left(x_{21}, v_{2} \mid 0, v_{1} ; t\right)\right]$. A particular role is played by the value of density $\mu$ at contact

$$
\mu\left(0^{+}, v_{2} \mid 0, v_{1} ; t\right)=\lim _{\substack{x \rightarrow 0 \\ x>0}} \mu\left(x, v_{2} \mid 0, v_{1} ; t\right)
$$

The notation $0^{+}$stresses the fact that the distance between the particles which are to collide approaches zero through positive values. The higher order conditional distributions $\mu_{s}(2,3, \ldots, s \mid 1 ; t), s=3,4, \ldots$ factorize for all times $t>0$ as

$$
\mu_{s}(2,3, \ldots, s \mid 1 ; t)=\prod_{j=2}^{s} \mu(j \mid j-1 ; t), \quad s=3,4, \ldots
$$

providing that this condition is fulfilled at time $t=0$. As a consequence, $\mu(2 \mid 1 ; t)$ has the extraordinary property to obey exactly the closed nonlinear integro-differential equation (3).

As we shall see later, it will be more convenient to consider $\nu\left(x_{2}, v_{2} \mid x_{1}, v_{1} ; t\right) \equiv \nu(2 \mid 1 ; t)$ the (conditional) density of particles with velocity $v_{2}$, which are at a distance $x_{2}-x_{1}$ of a given particle of velocity $v_{1}$. With Eq. (6) it follows that $\nu(2 \mid 1 ; t)$ can be expressed as:

$$
\nu(2 \mid 1 ; t)=\mu(2 \mid 1 ; t)+\sum_{s=3}^{\infty} \int d 3 \ldots \int d s \mu(2 \mid s ; t) \mu(s \mid s-1 ; t) \ldots \mu(3 \mid 1 ; t) .
$$

We can then write a new closed equation governing the dynamics of $\nu$ which, for a translationary invariant system, takes the form:

$$
\begin{aligned}
& {\left[\frac{\partial}{\partial t}+v_{21} \frac{\partial}{\partial x}+v_{21} \theta\left(v_{12}\right) \delta(x)\right] \nu\left(x, v_{2} \mid 0, v_{1} ; t\right)} \\
& =-\int d v_{3}\left\{\theta\left(v_{13}\right) v_{13} \nu\left(0^{+}, v_{3} \mid 0, v_{1} ; t\right)\left[\nu\left(x, v_{2} \mid 0, v_{3} ; t\right)-\nu\left(x, v_{2} \mid 0, v 1 ; t\right)\right]\right. \\
& +\theta\left(v_{23}\right) v_{23} \nu\left(0^{+}, v_{3} \mid 0, v_{2} ; t\right) \nu\left(x, v_{2} \mid 0, v 1 ; t\right) \\
& \left.+\theta\left(v_{32}\right) v_{32} \nu\left(0^{+}, v_{3} \mid 0, v_{1} ; t\right) \nu\left(x, v_{2} \mid 0, v 3 ; t\right)\right\} \text {. }
\end{aligned}
$$


A complete description of the dynamic of the system is obtained once the equation of motion for $f(v ; t)$, the density of particles with a velocity $v$, is given. It is readily obtained from the dynamics of ballistic annihilation. One finds:

$$
\begin{aligned}
\frac{\partial}{\partial t} f\left(v_{1} ; t\right) & \\
= & -\int d v_{2} \theta\left(v_{12}\right) v_{12} \nu\left(0^{+}, v_{2} \mid 0, v_{1} ; t\right) f\left(v_{1} ; t\right) \\
& -\int d v_{2} \theta\left(v_{21}\right) v_{21} \nu\left(0^{+}, v_{1} \mid 0, v_{2} ; t\right) f\left(v_{2} ; t\right) .
\end{aligned}
$$

For a symmetric initial condition [i.e. $\phi(v)=\phi(-v)$ ], this last equation takes a simplified form

$$
\begin{aligned}
\frac{\partial}{\partial t} f\left(v_{1} ; t\right)= & -f\left(v_{1} ; t\right) \int d v_{2}\left[\theta\left(v_{12}\right) v_{12} \nu\left(0^{+}, v_{2} \mid 0, v_{1} ; t\right)\right. \\
& \left.+\theta\left(v_{21}\right) v_{21} \nu\left(0^{+},-v_{2} \mid 0,-v_{1} ; t\right)\right]
\end{aligned}
$$

where we used that for a symmetric distribution

$$
f\left(v_{1} ; t\right) \nu\left(x, v_{2} \mid 0, v_{1} ; t\right)=f\left(-v_{2} ; t\right) \nu\left(x,-v_{1} \mid 0,-v_{2} ; t\right) .
$$

To get a closed analytical form for the particle density $f(v ; t)$, we thus have to solve Eq. (8), or at least to obtain the density at contact $\nu\left(0^{+}, v_{2} \mid 0, v_{1} ; t\right)$. This is a very difficult problem and despite numerous attempts, we have not yet been able to complete this program. Accordingly, we choose a less ambitious approach. In the next section, we shall propose a scaling theory for $f(v ; t)$ and $\nu\left(0^{+}, v_{2} \mid 0, v_{1} ; t\right)$ whose validity will be confirmed by numerical simulations.

\section{SCALING THEORY}

We are mainly interested in the behavior of the system in the long time limit, where the time dependence of the physical quantities of interest is given by power laws. Thus, it is natural to try to develop for them a dynamical scaling theory. The validity of this scaling theory will be attested a posteriori by numerical simulations.

For simplicity, we shall restrict our analysis to symmetric distributions. For dimensional reasons, a time dependent characteristic velocity should be introduced. For a symmetric distribution, the average velocity vanishes for all times. However, the mean-square velocity, denoted $M(t)$, does not vanish. Thus we shall assume that $[M(t)]^{1 / 2}$ is the time dependent characteristic velocity which will enter into the scaling theory.

Hence, we postulate the two following scaling laws:

$$
\begin{aligned}
& f(v ; t)=\frac{n(t)}{[M(t)]^{1 / 2}} \Psi\left(v[M(t)]^{-1 / 2}\right) \\
& \nu\left(0^{+}, v_{2} \mid 0, v_{1} ; t\right)=\frac{n(t)}{[M(t)]^{1 / 2}} \\
& \quad \times \Phi\left(0^{+}, v_{2}[M(t)]^{-1 / 2} \mid 0, v_{1}[M(t)]^{-1 / 2}\right),
\end{aligned}
$$

where $n(t)$ is the particle density at time $t$ :

$$
n(t)=\int d v f(v ; t)
$$

and

$$
M(t)=\frac{\int d v v^{2} f(v ; t)}{\int d v f(v ; t)} .
$$

It is useful to introduce the intermediate quantity [see Eq. (9)]:

$$
\begin{aligned}
I_{\mathrm{c}}\left(v_{1} ; t\right)=\int d v_{2}\left[\theta\left(v_{12}\right) v_{12} \nu\left(0^{+}, v_{2} \mid 0, v_{1} ; t\right)\right. \\
\left.+\theta\left(v_{21}\right) v_{21} \nu\left(0^{+},-v_{2} \mid 0,-v_{1} ; t\right)\right] .
\end{aligned}
$$

Using the scaling forms (11) and (12), one can write:

$$
\begin{aligned}
I_{\mathrm{c}}\left(v_{1} ; t\right)= & n(t)[M(t)]^{1 / 2}\left[\int d u_{2} \theta\left(u_{12}\right) u_{12} \Phi\left(0^{+}, u_{2} \mid 0, u_{1}\right)\right. \\
& \left.+\int d u_{2} \theta\left(u_{21}\right) u_{21} \Phi\left(0^{+},-u_{2} \mid 0,-u_{1}\right)\right] \\
\equiv & n(t)[M(t)]^{1 / 2} J_{\mathrm{c}}\left(u_{1}\right),
\end{aligned}
$$

where $u_{i}=v_{i}[M(t)]^{-1 / 2},(i=1,2)$. The time derivative of the density and the mean square velocity can be written as:

$$
\begin{aligned}
\frac{\dot{n}(t)}{n(t)} & =-\frac{\int d v I_{\mathrm{c}}(v ; t) f(v ; t)}{\int d v f(v ; t)} \\
& =-n(t)[M(t)]^{1 / 2} \frac{\int d u J_{\mathrm{c}}(u) \Psi(u)}{\int d u \Psi(u)} \\
\frac{\dot{M}(t)}{M(t)}+\frac{\dot{n}(t)}{n(t)} & =-\frac{\int d v v^{2} I_{\mathrm{c}}(v ; t) f(v ; t)}{\int d v v^{2} f(v ; t)} \\
& =-n(t)[M(t)]^{1 / 2} \frac{\int d u u^{2} J_{\mathrm{c}}(u) \Psi(u)}{\int d u u^{2} \Psi(u)} .
\end{aligned}
$$

We note that $\int d u J_{\mathrm{c}}(u) \Psi(u) / \int d u \Psi(u)$ and $\int d u u^{2} J_{\mathrm{c}}(u)$ $\times \Psi(u) / \int d u u^{2} \Psi(u)$ are two constants called $A$ and $B$ respectively. In the long time limit, the solution of Eqs (17) and (18) is:

$$
\begin{aligned}
n(t) & \sim t^{-\alpha} \\
M(t) & \sim t^{-2 \beta}
\end{aligned}
$$

with

$$
\begin{aligned}
& \alpha=2 A /(A+B) \\
& \beta=(B-A) /(A+B) .
\end{aligned}
$$

For all values of $A$ and $B$, the scaling law:

$$
\alpha+\beta=1
$$

holds. Note that this scaling law has already been obtained by Ben-Naim et al. [3] from heuristic arguments.

Using this scaling law, it is possible to justify a posteriori our scaling postulate for $\nu$. Indeed, let us introduce the dimensionless correlation function $g\left(x, v_{2}, v_{1} ; t\right)$ satisfying

$$
\nu\left(x, v_{2} \mid 0, v_{1} ; t\right)=g\left(x, v_{2}, v_{1} ; t\right) f\left(v_{2} ; t\right) .
$$



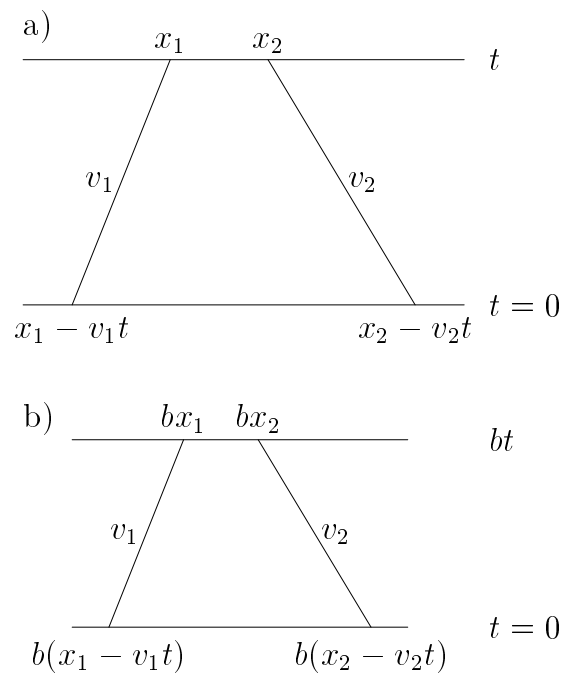

FIG. 1. Illustration of the similarity relation satisfied by the correlation function $g\left(x_{21}, v_{2}, v_{1} ; t\right)$. a) A typical two-particle configuration is shown at time $t$. Initially the positions of the particles are $x_{1}-v_{1} t$ and $x_{2}-v_{2} t$. They move respectively with velocity $v_{1}$ and $v_{2}$. b Same configuration in which time and distances have been rescaled by a factor $b$ (the velocities remaining unchanged). The trapezoid defined by the points $b\left(x_{1}-v_{1} t\right), b\left(x_{2}-v_{2} t\right)$ at $t=0$, and $b x_{2}, b x_{1}$ at $b t$ in Fig. b) is similar to the one defined by the points $x_{1}-v_{1} t, x_{2}-v_{2} t$ at $t=0$, and $x_{2}, x_{1}$ at $t$ in Fig a).

As shown on Fig. 1, by rescaling both length and time by a factor $b, g$ should satisfy the following similarity relation:

$$
g\left(x, v_{2}, v_{1} ; t\right)=g\left(b x, v_{2}, v_{1} ; b t\right) .
$$

Moreover, rescaling the velocities by a factor $c$ corresponds to a dilatation of the time scale by the same factor:

$$
g\left(x, c v_{2}, c v_{1} ; t\right)=g\left(x, v_{2}, v_{1} ; c t\right) .
$$

Combining these two relations and putting $b=n(t)$ and $c=[M(t)]^{1 / 2}$, one immediately finds

$$
\begin{aligned}
g\left(x, v_{2}, v_{1} ; t\right)=g( & n(t) x, v_{2}[M(t)]^{-1 / 2}, \\
& \left.v_{1}[M(t)]^{-1 / 2} ; n(t)[M(t)]^{1 / 2} t\right) .
\end{aligned}
$$

For sufficiently long time, the scaling law (23) implies that $n(t)[M(t)]^{1 / 2} \sim t^{-1}$. Using Eq. (24) and the scaling form (11) for $f$, one recovers then our scaling postulate (12). This confirms the self-consistency of our theory. Furthermore by introducing the scaling postulates (11, 12) in (10) one obtains:

$$
\begin{aligned}
\frac{\partial}{\partial t} f(v ; t) & =-\left[\frac{3 A-B}{2} \Psi(u)-\frac{B-A}{2} u \Psi^{\prime}(u)\right][n(t)]^{2} \\
& =-[n(t)]^{2} J_{\mathrm{c}}(u) \Psi(u),
\end{aligned}
$$

hence an expression relating $\Psi(u)$ to $J_{\mathrm{c}}(u)$ :

$$
\frac{3 A-B}{2}-\frac{B-A}{2} u \frac{\Psi^{\prime}(u)}{\Psi(u)}=J_{\mathrm{c}}(u) .
$$

Let us now suppose that, initially, $f(v ; 0) \sim|v|^{\mu} \theta\left(v_{0}-\right.$ $|v|)$, with $-1<\mu<0$. In this case Ben-Naim et al. [3] argue that the long time behavior of $n(t)$ is nonuniversal ( $\mu$-dependent) and that $\Psi(u)$ retains the same power-law dependence than $f$; in particular it diverges like $|u|^{\mu}$ for $u \rightarrow 0$. What could we say about this case within our scaling approach? Letting $u \rightarrow 0$ in Eq. (27), we find

$$
\frac{3 A-B}{2}-\frac{B-A}{2} \mu=J_{\mathrm{c}}(0),
$$

or in terms of the exponent $\alpha$ :

$$
\frac{2 \alpha-1}{1-\alpha}=\mu+\frac{2 J_{\mathrm{c}}(0)}{B-A} .
$$

$J_{\mathrm{c}}(0)$ might in principle depend on $\mu$. However, it is very unlikely that its dependence will be such as to exactly compensate the linear term in $\mu$, hence the nonuniversality of the exponent $\alpha$. On the contrary, if $\Psi(u)$ satisfies

$$
\lim _{u \rightarrow 0} u \frac{\Psi^{\prime}(u)}{\Psi(u)}=0
$$

[which is the case if $\Psi(u)$ and $\Psi^{\prime}(u)$ are continuous functions at $u=0$ and if $\Psi(0) \neq 0]$, we find that

$$
\frac{2 \alpha-1}{1-\alpha}=\frac{2 J_{\mathrm{c}}(0)}{B-A} \text {. }
$$

Hence $\alpha$ will be universal only if the right hand side does not depend on the details of the initial distribution. We are unfortunately unable to prove analytically this independence on the initial conditions. However, in the next section we shall show that this property is supported by precise numerical simulations for several initial velocity distributions. Furthermore we shall find on the same footing that $\Psi(u)$ is an universal Gaussian function. Note that for $\Psi(u)$ to be universal, one should have according to Eqs (27) and (31) that $J_{\mathrm{c}}(u) /(B-A)$ is universal.

\section{NUMERICAL SIMULATIONS}

To test the validity of our scaling forms (11) and (12), we have performed numerical simulations in one dimension. The method we used is an exact synchronous time evolution whose algorithm is detailed in Appendix. We considered the following three different initial continuous velocity distributions:

i) A Gaussian distribution:

$$
\phi(v)=\frac{1}{\sqrt{2 \pi} v_{0}} \exp \left(-\frac{v^{2}}{2 v_{0}^{2}}\right),
$$

with $v_{0}=[M(0)]^{1 / 2}=1$.

ii) A uniform distribution with a cut-off:

$$
\phi(v)=\frac{1}{2 v_{0}} \theta\left(v_{0}-|v|\right),
$$

with $v_{0}=\sqrt{3}$, such that $M(0)=1$; 
iii) A Lorentzian distribution

$$
\phi(v)=\frac{1}{\pi} \frac{v_{0}}{v_{0}^{2}+v^{2}},
$$

with characteristic velocity $v_{0}=1$.

For each simulation, we started with $2^{18}$ particles on a periodic chain of length 2 11]. Note that the initial characteristic collision time $\tau_{\mathrm{c}}=\left[n(0) v_{0}\right]^{-1}$ is of the order $10^{-5}$. We choose to distribute the particles uniformly on the line according to a Poisson law. However we verified that a regular spacing distribution does not modify the long time dynamics of the system.

The time dependent velocity distribution has been monitored yielding the following results. First, we plotted on a double logarithmic scale the number of particles (respectively the root mean square velocity of a particle) as a function of time. The exponents $\alpha$ (respectively $\beta$ ) are then extracted from the slopes of the curves. Linear regressions were made for various sets of points. The retained value is the one corresponding to the best fit and the error is given by the maxinum deviation. Typically, the fits were made for time intervals such that $10^{-3} \lesssim t \lesssim 1$. For longer times, the system starts to feel the boundary conditions. The lower limit of the time interval corresponds approximately to the beginning of the linear regime. Remark however that at this time about $98 \%$ of the particles have already disapeared. The results are given in Table 1 and Figures 2 . Data were averaged over $10^{4}$ samples for the Gaussian distribution and $10^{3}$ for the uniform and Lorentzian cases, respectively.

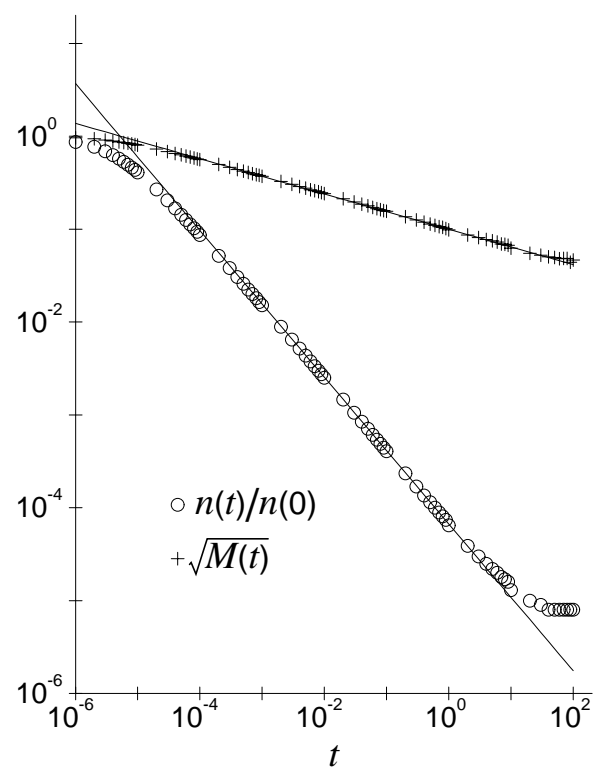

FIG. 2. Plot of the relative density (circle) and the root mean square velocity (plus) as a function of time, for a Gaussian initial velocity distribution $\phi(v)=\exp \left(-v^{2} / 2\right) / \sqrt{2 \pi}$. The periodic chain of length 2 initially contained $2^{18}$ particles. Data are averaged over $10^{4}$ samples. The two straight lines are obtained by linear regression over a subset of points (typically for $t$ between $10^{-3}$ and 1 ).
TABLE I. Numerical values of the exponents $\alpha$ and $\beta$ obtained from the simulations (see Fig. 2 (4). The error is \pm 0.005 for each value of $\alpha$ and $\beta$. Results are given for three different initial velocity distributions.

\begin{tabular}{cccc}
\hline \hline Velocity distribution & $\alpha$ & $\beta$ & $\alpha+\beta$ \\
\hline Gaussian & 0.785 & 0.195 & 0.980 \\
Uniform & 0.795 & 0.195 & 0.990 \\
Lorentzian & 0.780 & 0.195 & 0.975 \\
\hline \hline
\end{tabular}

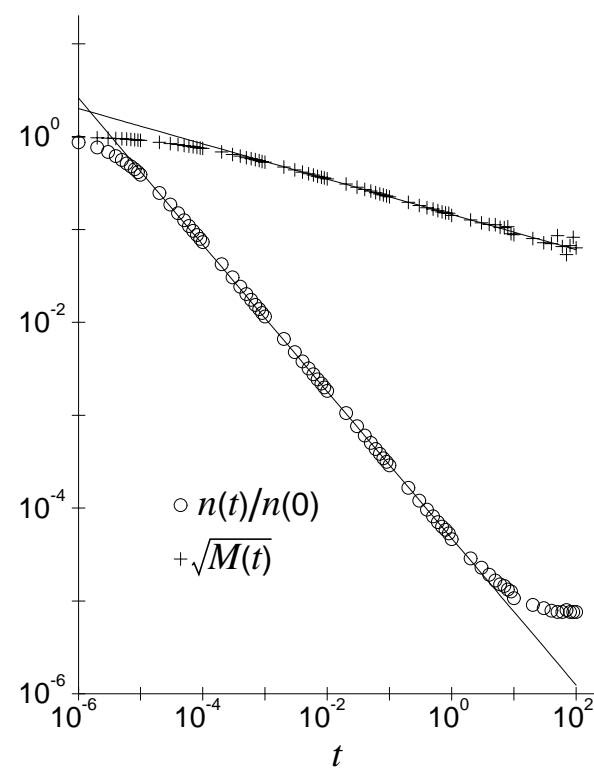

FIG. 3. Plot of the relative density (circle) and the root mean square velocity (plus) as a function of time, for a uniform initial velocity distribution $\phi(v)=\theta(\sqrt{3}-|v|) / \sqrt{12}$, so that $M(0)=1$. The periodic chain of length 2 initially contained $2^{18}$ particles. Data are averaged over $10^{3}$ samples. The two straight lines are obtained by linear regression over a subset of points (typically for $t$ between $10^{-3}$ and 1 ).

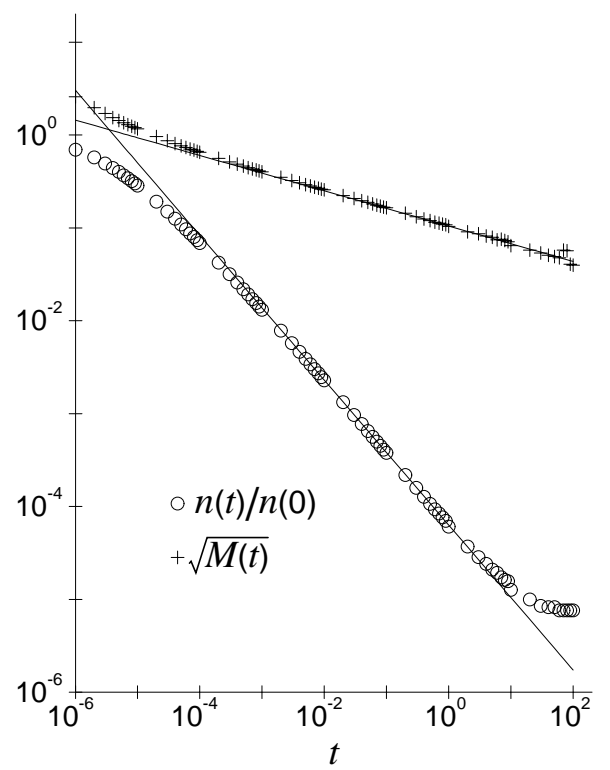

FIG. 4. Same as in Fig. 3 for a Lorentzian initial velocity distribution $\phi(v)=\pi^{-1}\left(1+v^{2}\right)^{-1}$. 
We see that the scaling law $\alpha+\beta=1$ is well satisfied for the different distributions. For the Lorentzian one however, the results are less precise. This is due to the fact that the scaling regime sets in for longer times, and longer simulations would be needed to reach the same precision.

Note that for a uniform velocity distribution, BenNaim et al. [3] obtained $\alpha=0.76$ and $\beta=0.22$, but with a slightly different algorithm (which involved diffusion in addition to the ballistic motion) and a poorer statistics.

From the values quoted in Table If we immediately deduce, at least for the three different velocity distributions considered here, that the exponents $\alpha$ and $\beta$ are the same. In view of the arguments presented in Section III, we are led to conjecture that there is universality for a wide class of initial conditions. However, this universality should not hold for initial distributions which diverge or vanish at $v=0$ [see Eq. (30)].

The scaling functions $\Psi(u)$ have also been measured for the three different initial distributions. In Figs 57 , the scaling functions $\Psi(u)$ are plotted versus the reduced velocity $u=v[M(t)]^{-1 / 2}$. For the Gaussian case, one sees (Fig. 5i) that $\Psi$ keeps its Gaussian shape until $t \leq 0.1$. In fact a finer analysis of the six first even moments of $\Psi\left(\left\langle u^{2 n}\right\rangle\right.$ with $\left.n=0,1, \ldots, 5\right)$ shows that $\Psi$ looses its Gaussian character when $t \gtrsim 0.2$, i.e. when less than 50 particles remain in the system. Ultimately, when less than 10 particles remain in the system, $\Psi$ tends to a bimodal distribution. We emphasize again that this late stage behavior is an artifact of the finiteness of the system. In the thermodynamic limit, the true asymptotic behavior would be Gaussian like. Similar conclusions can be drawn for the uniform and Lorentzian cases (see Figs 6 and (7). Indeed, after a transient regime, $\Psi$ adopts a Gaussian profile until $t \gtrsim 0.2$. These conclusions are

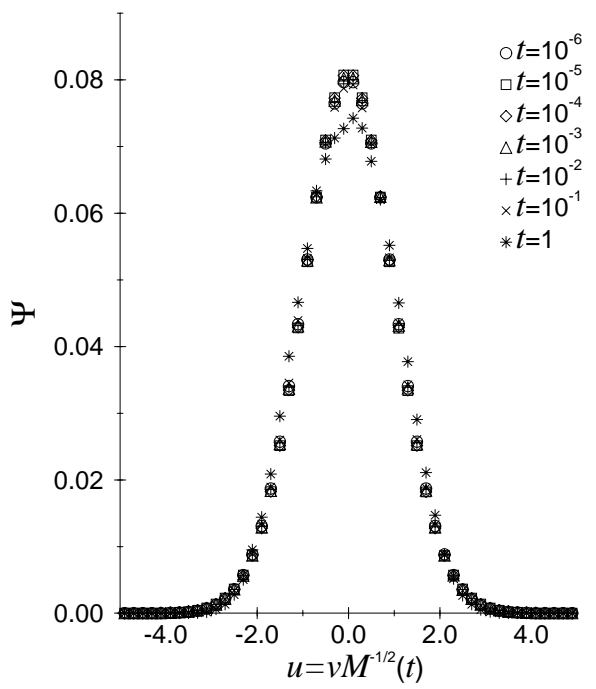

FIG. 5. Plot of the scaling function $\Psi$ for a Gaussian initial velocity distribution (see Fig. 2) as a fonction of the reduced velocity $u=v[M(t)]^{-1 / 2}$ for 7 different times. Data are averaged over $10^{4}$ samples. One remark that $\Psi$ keeps its Gaussian character as long as $t \leq 1$. again confirmed by the analysis of the moments. Thus, for these three distributions, we conclude that beside the universal behavior of the exponent, the scaling functions $\Psi(u)$ are also the same. There is an attractive Gaussian like scaling distribution in the long time regime:

$$
\Psi(u)=\frac{1}{\sqrt{2 \pi}} \exp \left(-u^{2} / 2\right) .
$$

Here again, we are led to conjecture that such a behavior would be valid for any velocity distribution which takes a finite non-zero value at $v=0$ and which is regular near $v=0$.

In addition, the range of validity of our scaling postulates can be tested. Indeed from Eqs (17,18), one sees that

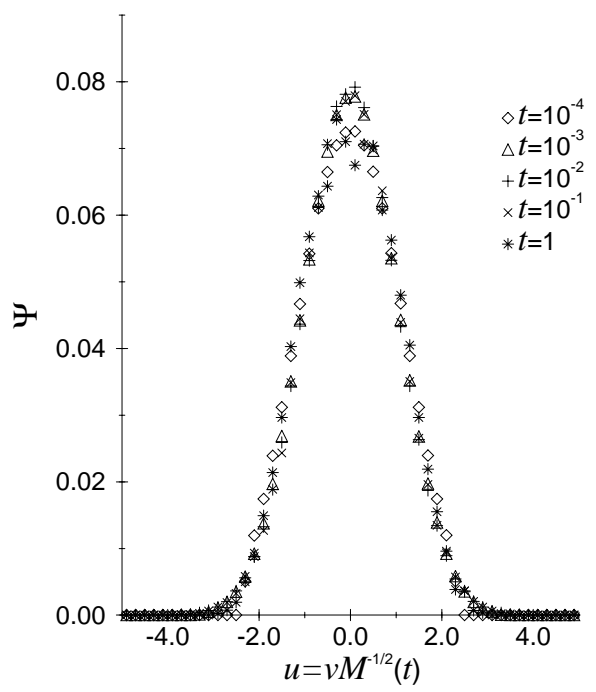

FIG. 6. Plot of the scaling function $\Psi$ for a uniform initial velocity distribution (see Fig. 3) as a fonction of the reduced velocity $u=v[M(t)]^{-1 / 2}$ for 5 different times. Data are averaged over $10^{3}$ samples. One remarks that after a transient regime not represented here, $\Psi$ is attracted towards a Gaussian like scaling distribution (as soon as $t \gtrsim 10^{-4}$ ).

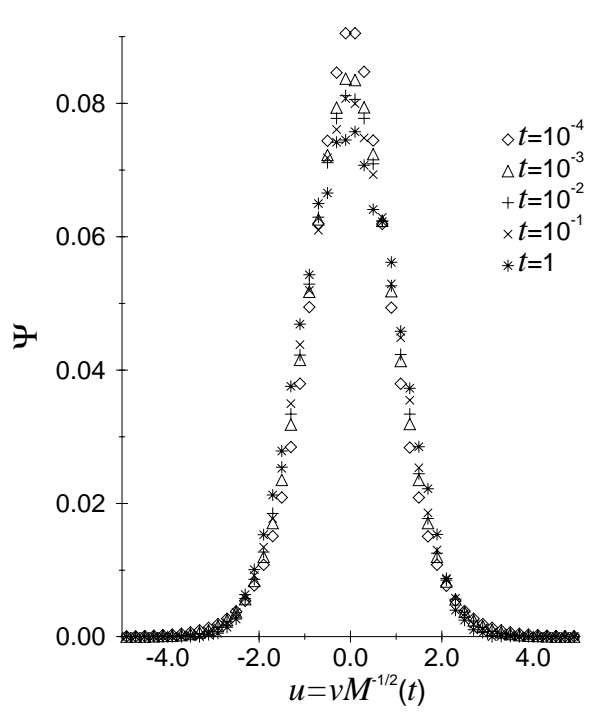

FIG. 7. Same as in Fig. 6 for a Lorentzian initial velocity distribution. 


$$
n(t)=\text { const }\left(n(t)[M(t)]^{1 / 2}\right)^{\alpha},
$$

so that the $\log -\log$ plot of $n(t)$ versus $n(t)[M(t)]^{1 / 2}$ should give a straight line. In Fig. 8 we reproduce this plot for a Gaussian initial distribution. We remark that Eq. (36) is satisfied for times as short as $t \simeq 10^{-6}$ (at this time less that $20 \%$ of the particles have already reacted) and as long as $t \simeq 5$. Note that the slope (i.e. the exponent $\alpha$ ) obtained by linear regression is slightly larger than the value obtained from Fig. 2. This comes from the fact that the sum $\alpha+\beta$ given by the value of the exponents measured on Fig. 2 gives 0.980 rather than strictly 1. Multiplying the slope of Fig. 8 by 0.980 reproduces indeed the value of $\alpha$ quoted in Table $\mathbb{1}$. For the uniform and Lorentzian distributions the range of validity of the scaling postulates is smaller, beginning only near $t \simeq 10^{-3}$. This fact confirms the particular role played by the Gaussian distribution in this problem.

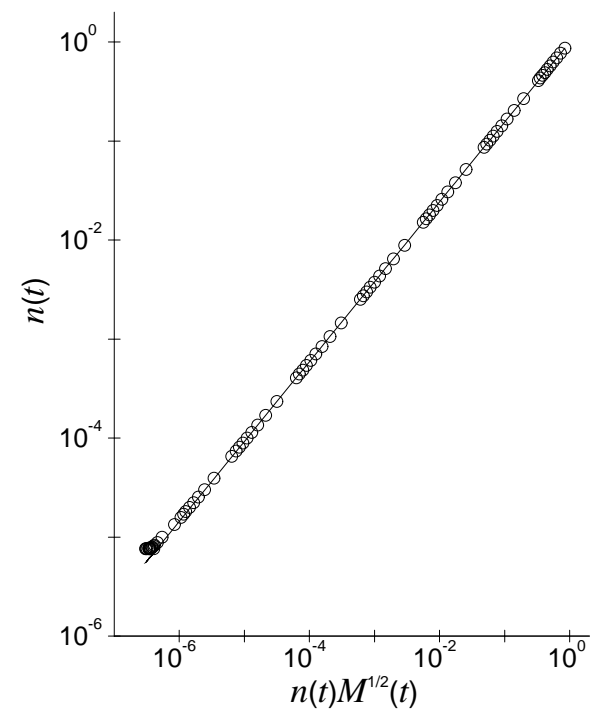

FIG. 8. Log-log plot of the density as a function of $n(t)[M(t)]^{1 / 2}$ for a Gaussian initial velocity distribution (data are the same than Fig. 2, note that large values of $n(t)[M(t)]^{1 / 2}$ correspond to short time). The comparison with a straight line is excellent both for short and long times. This shows that the range of validity of our scaling postulate is very wide for a Gaussian initial condition.

\section{CONCLUDING REMARKS}

We have studied the kinetics of ballistic annihilation for a one-dimensional ideal gas with continuous velocity distribution. Starting from an exact analytical approach previously derived, we established a scaling theory for the long time behavior of such systems. The validity of this scaling theory has been tested numerically for three different initial continuous velocity distributions (Gaussian, uniform and Lorentzian). Both the dynamical exponents and the scaling functions are the same for the three cases. This led us to conjecture that all the continuous velocity distributions which take a finite nonzero value at $v=0$ and which are regular near $v=0$ are attracted towards a Gaussian distribution and thus belong to the same universality class. Despite several attempts we have not yet been able to prove this conjecture in the framework of our exact analytical approach. We have only shown that a Gaussian distribution is compatible, in the long time regime, with the exact dynamical equation (8). However, we have not been able to prove that this Gaussian distribution was the only possible solution.

\section{ACKNOWLEDGMENTS}

Works partially supported by the Swiss National Science Foundation. One of us (J.P.) acknowledged the hospitality at the Department of Theoretical Physics of the University of Geneva were part of this work was done, and the financial support by KBN (Committee for Scientific Research, Poland) grant 2 P03 B 03512.

\section{APPENDIX: NUMERICAL ALGORITHM}

To simulate the ballistic annihilation in one dimension, the simplest algorithm is probably the standard molecular dynamics: starting from a given configuration, one identifies the shortest collision time, removes the two colliding particles and calculates the new positions of the remaining particles at this time. Starting from this new configuration, the process is iterated. This algorithm is very simple but not very efficient, the computing time increasing with the number of particles $N$ as $\mathcal{O}\left(N^{2}\right)$.

The numerical algorithm we used instead has been largely inspired by the one developed by Krapivsky et al. 4. The idea is to establish the list of all the ("true") collision times arranged in chronological order. From the initial condition we compute the collision times of each particle with its right nearest neighbor and sort those times into an ascending series, called $\mathcal{A}$, using a standard sorting algorithm (see for example [12]). The shortest time of this set corresponds to the first "true" collision time and is the first member of the list of the "true collision times"; simultaneously it is removed from $\mathcal{A}$. Then one removes the pair of particles $(n, n+1)$ corresponding to this first collision. As a consequence, the collision times associated with the pairs $(n-1, n)$ and $(n+1, n+2)$ should be discarded from $\mathcal{A}$, producing a truncated sorted list called $\mathcal{A}^{\prime}$. The collision time of the new nearest neighbor pair $(n-1, n+2)$ is computed and is the first element of a new unsorted list, called $\mathcal{N}$. The process is then iterated starting with the sorted list $\mathcal{A}^{\prime}$ as long as its first element is shorter than the shortest element of $\mathcal{N}$. When this is no longer true, we merge both lists $\left(\mathcal{A}^{\prime}\right.$ and $\left.\mathcal{N}\right)$ into a new one which is sorted. This last list replace $\mathcal{A}$ and the process continues until at most one particle remains in the system. For the continuous velocity distribution we considered, this merging step between $\mathcal{A}^{\prime}$ and $\mathcal{N}$ occurs very rarely. For example, for a Gaussian initial velocity distribution, it takes place approximately hundred times for $2^{17}$ iterations of a system containing initially $2^{18}$ particles, and the whole simulation used about thirty seconds of CPU on a HP 9000 Serie 700 workstation. The computing time increases with the number of particles $N$ roughly as $\mathcal{O}\left(N^{5 / 4} \ln N\right)$. 
[1] Y. Elskens and H. L. Frisch, Phys. Rev. A 31, 3812 (1985).

[2] J. Krug and H. Spohn, Phys. Rev. A 38, 4271 (1988).

[3] E. Ben-Naim, S. Redner and F. Leyvraz, Phys. Rev. Lett. 70, 1890 (1993).

[4] P. L. Krapivsky, S. Redner and F. Leyvraz, Phys. Rev. E 51, 3977 (1995).

[5] J. Piasecki, Phys. Rev. E 51, 5535 (1995).

[6] M. Droz, P.-A. Rey, L. Frachebourg and J. Piasecki, Phys. Rev. Lett. 75, 160 (1995).

[7] M. Droz, P.-A. Rey, L. Frachebourg and J. Piasecki, Phys. Rev. E 51, 5541 (1995).

[8] J. Piasecki, P.-A. Rey and M. Droz, Physica A 229, 515
(1996).

[9] P.-A. Rey, M. Droz and J. Piasecki, Eur. J. Phys. 18, 213 (1997).

[10] J. Medhi, Stochastic Processes (John Wiley and Sons, New York, 1996).

[11] For open systems, the evolution does not notably differ as long as $t \lesssim 2$, the time needed by a particle of velocity $v_{0}$ to totally cross the system.

[12] W. H. Press, S. A. Teukolsky, W. T. Vetterling and B. P. Flannery, Numerical Recipes in Fortran, The Art of Scientific Computing (Cambridge University Press, Cambridge, 1992). 